\title{
Application of Hilbert Spectroscopy to Pulsed Far-Infrared Radiation
}

\author{
V. Shirotov, Y. Divin, U. Poppe, H. Larue, E. Zimmermann, A. Ahmet, H. Halling, and K. Urban
}

\begin{abstract}
We present our results on the development and characterization of a prototype of Hilbert spectrometer, which is intended to operate with pulsed far-infrared radiation. The new prototype consists of high- $T_{c}$ Josephson detector in an optical cryostat, analog electronics with the bandwidth of $14 \mathrm{MHz}$, and a DSP-based data acquisition system, controlling spectroscopic measurements. The specially developed digital data acquisition system gives a possibility to operate in two regimes-with and without integration of the pulsed signal. The tests have been carried out using pulsed $94 \mathrm{GHz}$ radiation with pulse duration of $200 \mathrm{~ns}$ and a pulse repetition rate of $1 \mathrm{MHz}$. A measuring time of $7 \mathrm{~ms}$ for a data set of 512 spectral points has been realized. It has been demonstrated, that in a broadband $(\approx 10 \mathrm{MHz})$ regime of measurements without integration the developed spectrometer has a dynamic range of external signal power of $17 \mathrm{~dB}$, which can be enhanced by using integration function.
\end{abstract}

Index Terms-Far infrared spectroscopy, high- $T_{c}$ Josephson detector, hilbert spectroscopy.

\section{INTRODUCTION}

$\mathbf{H}$ ILBERT spectroscopy is based on the ac Josephson effect [1] and for a long time, due to extremely short time constant of Josephson junctions [2], it has been considered as a good candidate for fast spectroscopic measurements. However, up to now, all experiments by this technique have been carried out in rather slow regimes.

It has been demonstrated that Hilbert spectroscopy can be reliably used in a very broadband frequency range from several gigahertz up to several terahertz [3]. An experimentally observed power dynamic range of the external signal was found to be around $50 \mathrm{~dB}$ [4]. Recently, it has been also demonstrated [5], that this technique could be sensitive enough to operate with a conventional broadband radiation source (high pressure mercury arc lamp), which had an extremely low spectral density in far infrared range. The usage of more powerful radiation sources for broadband experiments with Hilbert spectroscopy might lead to a better signal to noise ratio and to higher speed measurements.

In last decade, several new approaches have been developed for the generation of the intensive FIR radiation, e.g., photoconductive or electro-optical terahertz emitters excited

Manuscript received August 6, 2002.

V. Shirotov was with the Institute of Solid-State Research, Research Center Juelich, D-52425 Juelich, Germany. He is now with the Institute of Radioengineering and Electronics of RAS, Mokhovaya 11-7, Moscow 101999, Russia (e-mail: votoriw@mail.ru).

Y. Divin, U. Poppe and K. Urban are with the Institute of Solid-State Research, Research Center Juelich, D-52425 Juelich, Germany.

H. Larue, E. Zimmerman, A. Ahmet and H. Halling are with the Central Electronic Laboratory, Research Center Juelich, D-52425 Juelich, Germany.

Digital Object Identifier 10.1109/TASC.2003.813673 by femtosecond laser pulses [6], [7], germanium hot hole lasers [8] and coherent synchrotron or transition radiation from relativistic electron bunches [9], [10]. Essential features of these new sources are a high spectral density of radiation and the pulse operation with a repetition rate in megahertz range. These aspects give a principal possibility to increase the speed of spectroscopic experiments.

Hilbert spectroscopy based on high- $T_{c}$ selective Josephson detector has been already used for spectral measurements of coherent transition radiation [11]. These experiments have been carried out in a slow regime with data integration over around 10 seconds. The usage of the low-frequency electronics gave no possibility for the resolution of the fine time structure and for a performance of the fast measurements.

It is of interest to develop a Hilbert spectrometer, which would be able to operate with the pulsed radiation signals and could experimentally demonstrate advantages and potentials of fast Hilbert spectroscopy. At present, the speed limitations in Hilbert spectroscopy techniques come from the available analog and digital electronics. Thus, the application of this technique to pulsed radiation requires an enhancement of the frequency bandwidth of the analog electronics and the speed of the data acquisition.

Here, we present the results of the development and characterization of Hilbert spectrometer prototype for the operation with pulsed far-infrared radiation.

\section{EXPERIMENT}

\section{A. Prototype of Hilbert Spectrometer}

We have developed a new prototype of the Hilbert spectrometer consisting of an optical cryostat, a $\mathrm{YBa}_{2} \mathrm{Cu}_{3} \mathrm{O}_{7-x}$ grain-boundary Josephson junction as a frequency-selective detector, an analog electronics, a digital data acquisition and control system. A schematic view of this prototype without the digital electronics is shown in Fig. 1.

In this work we used grain-boundary $\mathrm{YBa}_{2} \mathrm{Cu}_{3} \mathrm{O}_{7-x}$ Josephson junctions made on $2 \times 14^{\circ} \mathrm{NdGaO}_{3}$ substrate [12]. The junction was placed on a temperature control stage in the optical cryostat. A broadband coupling of Josephson junction with external radiation has been realized with a help of broadband planar log-periodic silver antenna, sputtered above the junction on the same substrate, and a silicon hyperhemispherical lens, which concentrate the radiation and provide better coupling of the antenna with a free space.

Josephson junction operation as a frequency-selective detector was accomplished by an analog electronic scheme, which applies a dc current bias through the junction and amplifies 


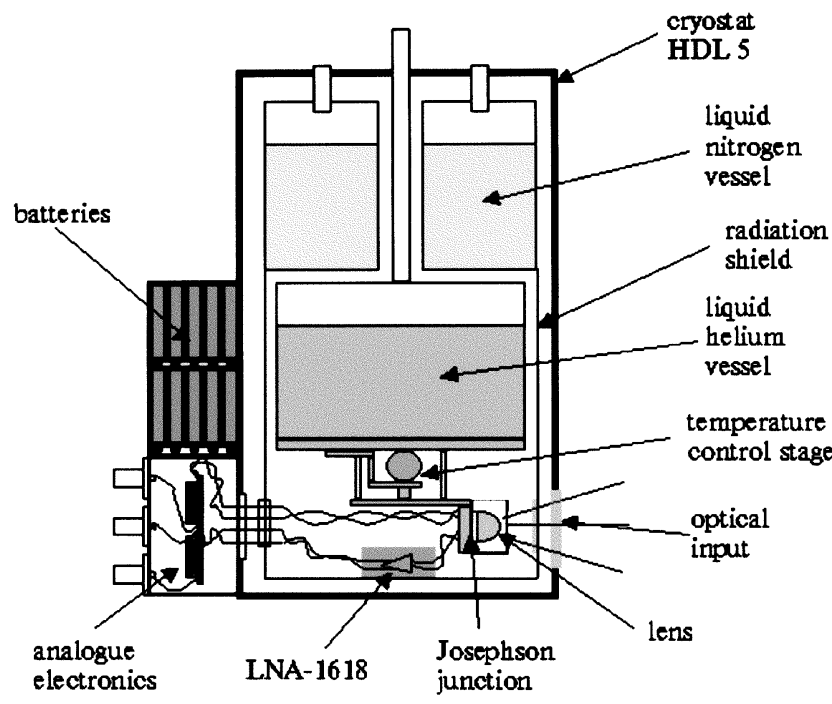

Fig. 1. Schematic view of the cryogenic and analog electronics parts of the new Hilbert spectrometer prototype.

signals from it. The main part of an amplifying part of this scheme was based on a cryogenic low-noise preamplifier LNA-1618 with noise level $0.16 \mathrm{nV} /(\mathrm{Hz})^{1 / 2}$ [13]. This analog scheme had its own battery power supply and its frequency bandwidth could be as large as $14 \mathrm{MHz}$.

A specific part of new Hilbert spectrometer prototype is its digital electronics, which included the experiment control and data acquisition systems. This new specially designed digital electronics differs this prototype from all ever reported before. The characteristic features of used hardware and software are an operation with pulsed signals and a possibility for an optimization of this process. This digital electronics represents a circuit, which includes digital-analog converter (DAC4813AP by Burr Brown) and analog-digital converters (ADC7810P by Burr Brown), and whose operation is based on a digital signal processor (DSP_-TMS320C32 by Texas Instruments, Incorporated) and a field programmable gate array (FPGA-XC4010 by XILINX).

According to the basic principals of the Hilbert spectroscopy technique [1], digital block supplies one control channel for a scanning of the dc bias on the Josephson junction and two recording channels for the measurements of the junction's dc characteristic ( $I-V$ curve) and its response to the external radiation $\left(\Delta I(V)=I(V)-I_{0}(V)\right.$,where $I(V)$ and $I_{0}(V)$ are $I-V$ curves of the junction with and without radiation correspondingly). The data acquisition system has also one, common for both measuring channels, reference input, signal on which starts the measuring process.

The developed digital electronic system has a special buffer for a temporal storage of measured data sets. This buffer is able to contain up to 512 data points. When the buffer is full, the system transfers measured data array to the control PC through a RS232 port within a $1 \mathrm{~s}$ time.

The control channel scans the dc signal with the rate of about $7 \cdot 10^{4}$ points per second. It determines the speed limitation for the experiment performance to around $7 \mathrm{~ms}$ for the whole data set. During this scanning, two recording channels take corre-

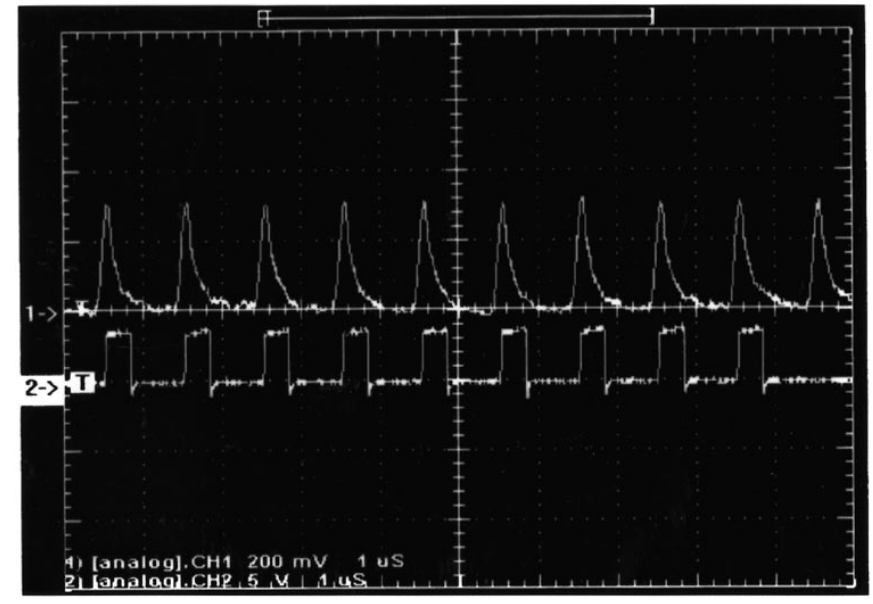

Fig. 2. Trace 1-time dependence of the signal from the Hilbert spectrometer prototype, irradiated by pulsed $94 \mathrm{GHz}$ radiation with $1 \mathrm{MHz}$ repetition rate and $200 \mathrm{~ns}$ pulse duration. Trace 2-example of possible complex integration window, consisting of nine simple ones.

sponding values in consecutive order (at constant biasing signal) and store them simultaneously.

The developed data acquisition system has several operational regimes. First, the input analog signals can be directly recorded by ADCs (ADC mode). Second, they can also be recorded using an integration function (Integrator mode) at every data point or averaging whole data arrays. Measurements control parameters can be chosen using the software, which illustrates the measuring process on a control PC as well.

The hardware gives the possibility for the operation in ADC mode (that is, in principal, also the integration on the ADC input) with a time parameter, characterizing the signal integration interval of $\tau=40 \mathrm{~ns}$. During this time the noise signal is averaged, and an effective frequency bandwidth of the analog signal measuring channel is $\Delta F=1 / 2 \tau=12.5 \mathrm{MHz}$. In the integrator mode every data point can be integrated over a simple integration window, characterizing by its start and end points, or over a complex integration window, consisting of several simple ones. The time duration of the integration window can be set with $40 \mathrm{~ns}$ time domain. If the integration is performed over the time interval $\tau_{i}$, it leads to the effective noise averaging over this time and to a decrease of its value by a factor of $\left(\tau_{i} / \tau\right)^{1 / 2}$. The data sampling rate for the ADCs in use is about $800 \mathrm{kHz}$.

\section{B. Experimental Set-Up}

The W-band has been chosen for the characterization of the new Hilbert spectrometer prototype. This is a highest frequency range where precision commercial attenuators and fast PINswitches are commercially available. An experimental set-up is similar to that one used in [14] for the characterization of the high- $T_{c}$ Josephson detector dynamic range. An essential difference is in the electronic blocks of Hilbert-spectrometers. As a source of the radiation we use a Gunn oscillator operating at $94 \mathrm{GHz}$. The modulation signal was taken from a pulse generator, which gave continuous pulse train with $1 \mathrm{MHz}$ repetition rate and $200 \mathrm{~ns}$ pulse duration. With this pulse repetition rate and the data sampling rate of used ADCs, every second pulse could be registered. 

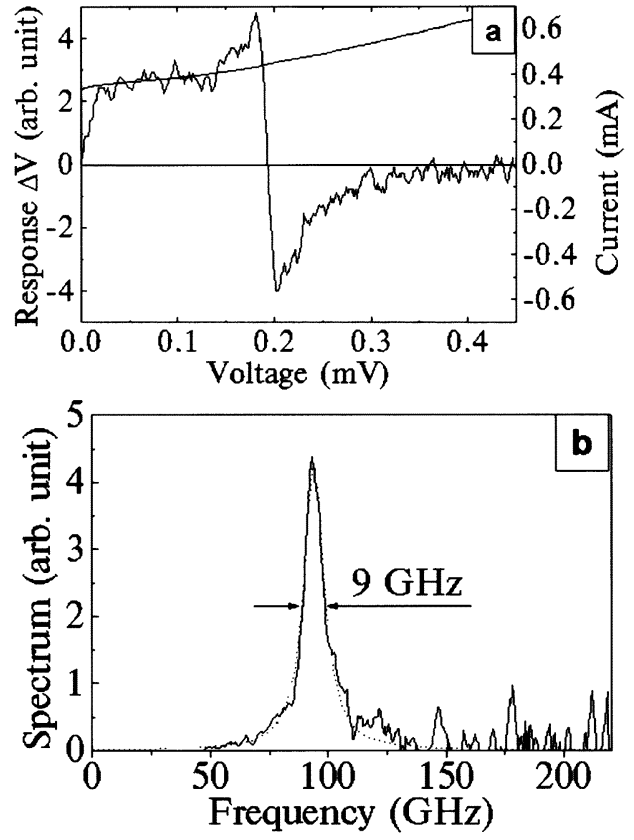

Fig. 3. (a) - measured response function and the $I-V$ curve of the selective Josephson detector. (b) - spectrum of pulsed $94 \mathrm{GHz}$ radiation, recalculated according to Hilbert spectroscopy. The dot line is a lorentzian fit.

\section{RESULTS AND DISCUSSION}

A principal suitability of the analog electronic scheme for fast spectroscopic experiments with pulsed radiation can be seen in Fig. 2. The trace 1 in Fig. 2 represents the pulse signal from the irradiated Josephson detector, amplified by analog electronic scheme. This figure demonstrates a good reproducibility of the chosen external radiation time structure by the analog electronic part of the developed prototype. Trace 2 in Fig. 2 represents an example of the complex integration window, consisting of nine simple ones.

Electrical characteristics for the Josephson junction $\left(R_{n}=\right.$ $0.85 \mathrm{Ohm}, I_{c}=370 \mu \mathrm{A}$ at $\left.T=77 \mathrm{~K}\right)$, i.e., junction $I-V$ curve and its response, obtained when the Hilbert spectrometer prototype was irradiated by pulsed monochromatic signal are presented in Fig. 3(a). These results are similar to that ones obtained in slow regime with stationary signal [14]. The results of a spectrum transformation according to Hilbert spectroscopy theory and its lorentzian fit are shown in Fig. 3(b). This figure demonstrates good agreement of experimental results and theory prediction. An achieved spectral resolution of $9 \mathrm{GHz}$ is very close to theoretical estimation of that one for this experiment.

The results of the response amplitude measurements in two different regimes are summarized in Fig. 4. A power scale on these plots demonstrates the power of signal, which was induced by external radiation and interacted with Josephson junction. Solid lines indicate square-law behavior, which characterizes selective Josephson detector at low external signal level. Arrows show the noise level and a limit sensitivity at output signal to noise ratio equals to 1 . Fig. 4 also demonstrates a dynamic range of the external signal power, i.e., the external signal power range, in which a deviation of the selective Josephson detector behavior from the square law is less than $3 \mathrm{~dB}$.

As it can be seen in Fig. 4(a), the minimal external signal power registered in the ADC mode, equals to about
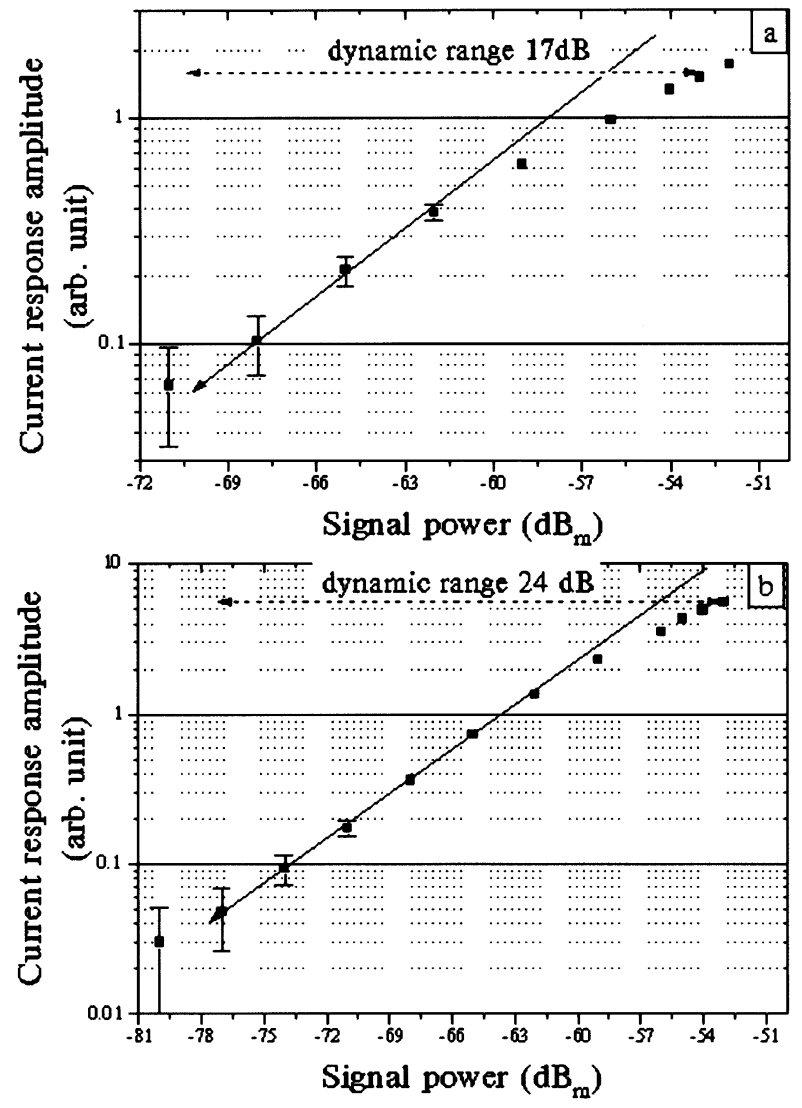

Fig. 4. Dependence of the current response amplitude of Josephson detector in selective regime versus external monochromatic signal power. (a) - results obtained in ADC mode; (b) — results obtained in integrator mode with using integration function over the complex integration window consisting of seven simple ones with time duration of about $280 \mathrm{~ns}$.

$1 \cdot 10^{-10} \mathrm{~W}(-70 \mathrm{dBm})$. The dynamic range in this regime is about $17 \mathrm{~dB}$ (signal power between -70 and $-53 \mathrm{dBm}$ ). This result is in the good agreement with dynamic range value, measured in slow regime with narrow bandwidth (around $1 \mathrm{~Hz}$ ) of the measuring channel [5]. Therefore, one should expect a drastic decrease of the dynamic range value with respect to observed in [5] $(50 \mathrm{~dB})$ when the bandwidth of the measurements increases up to about $10 \mathrm{MHz}$. Namely, the dynamic range value decrease has to be about $35 \mathrm{~dB}$. The experimentally observed $17 \mathrm{~dB}$ dynamic range is still a significant power range. It gives possibilities for the optimization in spectroscopic experiments with unknown radiation spectrum using Hilbert spectroscopy technique.

It is very important, that the low-noise cryogenic preamplifier (LNA-1618) has been used for the development of fast, broadband electronics. All commercially available operational preamplifiers [15], operating at room temperature, have about 10 times higher typical noise levels, and those usage would lead to 10 times higher value of minimal registered signal power and $10 \mathrm{~dB}$ less dynamic range. This fact could lead to significant problems, and it makes even impossible measurements in the regime described above.

The results of a dynamic range study in the integration mode are presented in Fig. 4(b). In this case, the integration over the complex window, consisting of 7 simple ones with the duration of about $280 \mathrm{~ns}$, has been used. This integration window con- 
figuration has been chosen in order to optimize the integration process of every data point over the time periods when the pulse signal is present and not to increase the whole data set measuring time, which is limited by the rate of control channel. The usage of the integration window, consisting of a higher number of the simple windows, as well as the usage of the whole data sets averaging function leads to an increase of the measuring time.

In described integration regime the effective time of each data point integration (noise smoothing) equals to $\tau_{i}=7 \times 280=$ $1960 \mathrm{~ns}$, and the significant decrease of about $7.5 \mathrm{~dB}$ of the minimal registered signal power in comparison with ADC mode can be easily observed in Fig. 4. This improvement of the sensitivity and the corresponding enlargement of the dynamic range are in a good agreement with a theoretical estimation of a system noise decrease, which gives a coefficient $\left(\tau_{i} / \tau\right)^{1 / 2}=7$. A minimal external signal power that can be registered in this regime equals to about $1.8 \cdot 10^{-11} \mathrm{~W}(-77.5 \mathrm{dBm})$.

System signal characteristics can be improved by implementation of the analog preamplifier with the noise level lower, than that of LNA-1618. In our case, the limit sensitivity is about $2 \div 3$ times worse than that one determined by the selective Josephson detector, because the preamplifier noise level exceeds the Josephson junction noise level.

To improve the parameters of the Hilbert spectrometer prototype, it would be also preferable to:

1) increase the scanning rate of the controlling channel;

2) use faster ADCs in the measuring channels;

3) enlarge the amount of the storing data points;

4) increase the rate of the data transferring system.

\section{CONCLUSIONS}

The Hilbert spectrometer prototype based on the high- $T_{c}$ Josephson junction for the operation with pulsed far-infrared radiation is developed and characterized.

The measuring time of this prototype is shown to be as small as $7 \mathrm{~ms}$ for 512 points data set. The power dynamic range of the external W-band signal in the ADC mode was $17 \mathrm{~dB}$ above the minimal power of $1 \cdot 10^{-10} \mathrm{~W}(-70 \mathrm{dBm})$ that can be registered with around $10 \mathrm{MHz}$ frequency bandwidth. The corresponding parameters in the optimized integrator mode were shown to be $24 \mathrm{~dB}$ and $1.8 \cdot 10^{-11} \mathrm{~W}(-77.5 \mathrm{dBm})$. Additionally, there is the possibility of these parameters improvement, increasing the measuring time and using the integration function over the larger pulse number or averaging the whole data sets.

The prototype of Hilbert spectrometer, described here, can be used not only in the W-band, where it was characterized at pulse operation mode, but also at higher frequencies up to $4 \mathrm{THz}$ [5]. However, similar characterization of this spectrometer in the pulse operation regime at subterahertz and, especially, at terahertz ranges is definitely a more difficult problem due to the lack of precision attenuators, fast switches, etc.

\section{ACKNOWLEDGMENT}

The authors are thankful to N. Ukhanskii for his help with a design of the analog electronics for the Hilbert spectrometer prototype.

\section{REFERENCES}

[1] Y. Y. Divin, O. Y. Polyanski, and A. Y. Shul'man, Sov. Techn. Phys. Lett., vol. 6, pp. 454-457, 1980.

[2] K. K. Likharev, Dynamics of Josephson Junctions and Circuits. New York: Gordon and Breach, 1986.

[3] Y. Y. Divin, O. Y. Volkov, M. V. Laytti, V. V. Shirotov, V. V. Pavlovskii, U. Poppe, P. M. Shadrin, and K. Urban, Physica C, vol. 372-376P1, pp. 422-425, 2002.

[4] V. V. Shirotov, Y. Y. Divin, and K. Urban, Physica C, vol. 372-376, p. $1,2002$.

[5] GermanyY. Divin, O. Volkov, V. Pavlovskii, V. Shirotov, P. Shadrin, U. Poppe, and K. Urban, Advances in Solid State Phys, K. Urban and B. Kramer, Eds. Berlin: Springer, 2001, vol. 41, p. 301.

[6] D. Grischkowsky, Frontiers in Nonlinear Optics, H. Walther, N. Koroteev, and M. O. Scully, Eds. Philadelphia, PA: Inst. of Phys., 1992.

[7] A. Nahata, A. S. Weiling, and T. F. Heinz, Appl. Phys. Lett., vol. 69, p. 2321, 1996.

[8] E. Gornik and A. A. Andronov, Opt. Quantum Electron., vol. 23, p. 111, 1991.

[9] T. Nakazato et al., Phys. Rev. Lett., vol. 63, p. 1245, 1989.

[10] U. Happek, A. J. Sievers, and E. B. Blum, Phys. Rev. Lett., vol. 67, p. 2962, 1991.

[11] Y. Y. Divin et al., IEEE Trans. Appl. Supercond., vol. 9, pp. 3346-3349, 1999.

[12] —, Proc. EUCAS, H. Rogalla and D. H. A. Blank, Eds., 1997, pp. 467-470.

[13] LNA-1618 Low-Noise, Liquid-Nitrogen Preamplifier, AGIV GROUP Limited, Moscow, Russia.

[14] V. Shirotov, Y. Divin, and K. Urban, IEEE Trans. Appl. Supercond., vol. 11, p. 955, Mar. 2001.

[15] Analog Devices Incorporated, Norwood, MA [Online]. Available: www.analog.com/technology/amplifiersLinear/precisionAmps/low NoisePA.html 